\title{
UNE REMARQUE SUR HYPOELLIPTICITÉ
}

\author{
YOSHIO KATO
}

\author{
dédié à Monsieur le Professeur K. Noshiro, à l'occasion \\ de son soixantième anniversaire
}

1. Un opérateur différentiel $P(x, D)$ à coefficients $C^{\infty}$ est dit hypoelliptique si toute solution de l'équation

$$
P(x, D) u=f
$$

est indéfiniment dérivable quand $f \in C^{\infty}$. $\Pi$ est bien connu qu'un opérateur formellement hypoelliptique est hypoelliptique (dont la démonstration a été donnée par plusieurs auteurs). D'autre part, Trèves [2] a introduit une condition suffisante sous laquelle un opérateur est hypoelliptique et qui englobe le cas formellement hypoelliptique (voir Définition 1). Ensuite, après une certaine date, Hörmander [1] a donné l'autre condition qui est strictement plus large que celle d'hypoellipticité formelle, eu égard au fait que la condition de Trèves est implicite et difficile à vérifier pour un opérateur donné (voir Définition 2). Cela nous a poussés à étudier la relation entre les condition suffisantes qu'ils établissent. Dans cet article on démontrera que la condition de Hörmonder est plus forte que celle de Trèves (voir notre théorème).

D'abord nous introduirons de certains espaces fonctionnels, avec Trèves [2] ( $N$ désignant l'ensemble des entiers $\geqslant 0)$ :

1) $A^{s} \ni f(s$ nombre réel $) \Leftrightarrow f \in \mathscr{S},|\hat{f}(\xi)|(1+|\xi|)^{s} \in L_{\xi}^{\infty 1)}$

$$
f_{n} \rightarrow 0\left(A^{s}\right)(n \rightarrow \infty) \Leftrightarrow N_{s}\left(f_{n}\right) \equiv \sup _{\xi \in R^{n}}\left|\hat{f}_{n}(\xi)\right|(1+|\xi|)^{s} \rightarrow 0 \quad(n \rightarrow \infty)
$$

$\left.1^{\prime}\right) \quad A_{\mathrm{loc}}^{s} \ni f \Leftrightarrow \varphi f \in A^{s}(\forall \varphi \in \mathscr{D})$

$$
f_{n} \rightarrow 0\left(A_{\mathrm{loc}}^{s}\right)(n \rightarrow \infty) \Leftrightarrow \varphi f_{n} \rightarrow 0\left(A^{s}\right)(n \rightarrow \infty)(\forall \varphi \in \mathscr{D})
$$

2) $A^{s, d} \exists f(s, d$ nombres réels $) \Leftrightarrow x^{\alpha} f \in A^{s+d^{\alpha} \mid} \mid\left(\forall \in N^{n}\right)$

$$
\left(x^{\alpha}=x_{1}^{\alpha_{1}} \ldots \ldots x_{n}{ }^{\alpha_{n}}, \quad|\alpha|=\alpha_{1}+\ldots \ldots+\alpha_{n}\right)
$$

Reçu le 30 juin, 1966.

1) Nous désignons par $\mathscr{S}$ l'espace des distributions tempérées sur $R^{n}$ et par $\hat{f}$ la transformée de Fourier de $f$. 


$$
f_{n} \rightarrow 0\left(A^{s, d}\right)(n \rightarrow \infty) \Leftrightarrow x^{\alpha} f_{n} \rightarrow 0\left(\left.A^{s+d}\right|^{\alpha} \mid\right)(n \rightarrow \infty)\left(\forall \alpha \in N^{n}\right)
$$

$\left.2^{\prime}\right) \quad A_{\mathrm{loc}}^{s, d} \ni f \Leftrightarrow \varphi f \in A^{s, d}(\forall \varphi \in \mathscr{D})$

$$
f_{n} \rightarrow 0\left(A_{10 c}^{s, d}\right)(n \rightarrow \infty) \Leftrightarrow \varphi f_{n} \rightarrow 0\left(A^{s, d}\right)(n \rightarrow \infty)(\forall \varphi \in \mathscr{D})
$$

3) Soit $\Omega$ un ensemble ouvert dans $R^{n}$, et $E$ un espace vectoriel topologique. Alors,

$\mathcal{E}_{y}(\Omega)(E) \ni f \Leftrightarrow f: \Omega \rightarrow E$ est indéfiniment différentiable, $\mathcal{E}_{y}^{p}(\Omega)(E) \ni f(p \in N) \Leftrightarrow f: \Omega \rightarrow E$ est $p$ fois continûment différentiable.

4) $\mathcal{E}_{y}(\Omega)\left(k, A^{s, d}\right) \ni f(s, d, k$ nombres réels $) \Leftrightarrow f \in \mathcal{E}_{y}^{p}(\Omega)\left(A^{s-k p, d}\right)(\forall p \in N)$.

$\left.4^{\prime}\right) \quad \mathcal{E}_{y}(\Omega)\left(k, A_{\mathrm{loc}}^{s, d}\right) \ni f \Leftrightarrow \varphi f \in \mathcal{E}_{y}(\Omega)\left(k, A^{s, d}\right)\left({ }^{\forall} \varphi \in \mathscr{D}\right)$

Soit $P(x, D)$ un opérateur différentiel qui est défini dans un ouvert $\Omega$ de $R^{n}$ et s'écrit sous la forme

$$
P(x, D)=\sum_{\alpha} a_{\alpha}(x) D^{\alpha}, \quad a_{\alpha}(x) \in C^{\infty}(\Omega) .
$$

où $\alpha \in N^{n}$ et $D^{\alpha}=\left(-i \partial / \partial x_{1}\right)^{\alpha_{1}} \ldots \ldots\left(-i \partial / \partial x_{n}\right)^{\alpha_{n}}$. Alors nous allons définir les critères d'hypoellipticité de [2] et de [1] là dessous.

Définition 1 (Trèves). Nous dirons que $P(x, D)$ est de type progressif sur $\Omega$ s'il existe une distribution $E(x, y)$ sur $R_{x}^{n}(y \in \Omega)$ telle que $P\left(y, D_{x}\right) E(x, y)=\delta(x)$ pour chaque $y \in \Omega$ et qui possède les propriétés suivantes:

(T. 1) Il existe des nombres réels $s, d$ et $k(d>0,0 \leqslant k<1)$ tels que $E(x, y) \in \varepsilon_{y}(\Omega)\left(k ; A_{\text {loc }}^{s, d}\right)$.

(T. 2) Pour tout ouvert borné $U, U \subset \bar{U} \subset \Omega$, il existe une fonction $\omega(x) \in \mathscr{D}$ de support contenu dans $\{x ; x+U \subset \Omega\}$, égale à 1 au voisinage de 0 , telle que des distributions $T_{j}(x, y)$ sur $R_{x}^{n}(y \in U, j=0,1,2, \ldots \ldots)$ données par

(1. 1) $\left\{\begin{array}{l}T_{0}(x, y)=E(x, y), \\ T_{j}(x, y)=E(x, y)_{(x)}^{*}\left[\omega(x)\left\{P\left(y, D_{x}\right)-P\left(x+y, D_{x}\right)\right\} T_{j-1}(x, y)\right], \quad(i=1,2, \ldots \ldots)\end{array}\right.$

vérifient la suivante: pour tout entier $q \geqslant 0$, il existe un entier $p \geqslant 0$ tel que $T_{p}(x, y) \in C^{q}\left(R_{x}^{n} \times U_{y}\right)$.

Définition 2 (Hörmander). l'opérateur $P(x, D)$ est dit satisfaire la condition HE dans $\Omega$ si

(H. 1) $\quad P(x, D)$ n'est identiquement nul dans aucune composante de $\Omega$.

(H. 2) Il existe des nombres $d, k(0<d \leqslant 1,0 \leqslant k<1)$ et des fonctions $M_{j}(x, \xi)$ $\operatorname{sur} \Omega_{x} \times R_{\xi}^{n}$ tels que pour tout $\alpha, \beta \in N^{n}$, 


$$
\left\{\begin{array}{l}
\left|D_{\xi}^{\alpha} D_{x}^{\beta} P(x, \xi)\right| \leqslant C_{\beta},{ }_{x}(1+|\xi|)^{-d \mid \alpha} \mid M^{\beta-\alpha}(x, \xi) \widetilde{P}(x, \xi), \quad \xi \in R^{n} \\
1 \leqslant M_{j}(x, \xi) \leqslant C_{x}(1+|\xi|)^{k}, \quad \xi \in R^{n}, \quad j=1,2, \ldots \ldots, n
\end{array}\right.
$$

ici $C_{\beta, x}$ et $C_{x}$ sont bornés quand $x$ parcourt des ensembles compacts de $\Omega$, et on a utilisé les notations:

$$
\tilde{P}(x, \xi)=\left(\sum_{\alpha}\left|P^{\alpha}(x, \xi)\right|^{2}\right)^{1 / 2}\left(P^{\alpha}(x, \xi)=D_{\xi}^{\alpha} P(x, \xi)\right), M^{\beta_{-\alpha}^{\alpha}}=M_{1}^{\beta_{1}-\alpha_{1}} \ldots \ldots M_{n}^{\beta_{n-\alpha}} .
$$

ThÉOR亡̀me. Soit $P(x, D)$ un opérateur différentiel à coefficients indéfiniment dérivables dans un ouvert $\Omega$ de $R^{n}$. Si $P(x, D)$ vérifies la condition $H E$ dans $\Omega$, il est de type progressif dans chaque ouvert dont l'adhérence est contenue dans $\Omega$ et compact.

Pour démontrer le théorème nous construirons dans le paragraphe 2 une solution élémentaire de $P\left(y, D_{x}\right)$ vérifiant la propriété (T. 1), et dans le dernier paragraphe nous montrerons que cette solution élémentaire jouit de la propriété (T. 2).

2. Lemma (Trèves). Soit $Q(x, D)$ un opérateur différentiel à coefficients continus dans un ouvert $\Omega$ de $R^{n}$ tel que $Q\left(y_{0}, \xi\right) \not \equiv 0$ par rapport $\grave{a} \xi$ pour chaque $y_{0} \in \Omega$, et $K$ un ensemble compact de $\Omega$. Alors il existe des nombres réels positifs $\eta$, a et $b$ tels que pour $y_{0} \in K$ et $\xi_{0} \in R^{n}$ il existe un $\theta_{0} \in R^{n}$ tel que

$$
b \widetilde{Q}(y, \xi) \leqslant \inf _{|z|=1}\left|Q\left(y, \xi+z \theta_{0}\right)\right|
$$

pour $\xi \in R^{n}$ et $y \in R^{n}$ vérifiant la suivante:

$$
\left|\xi-\xi_{0}\right| \leqslant \eta, \quad\left|y-y_{0}\right| \leqslant a \widetilde{Q}\left(y_{0}, \xi_{0}\right) /\left(1+\left|\xi_{0}\right|\right)^{m},
$$

où $z$ est un variable complex, et $m=\max _{x \in K} m_{x}\left(m_{x}=l\right.$ 'ordre de $Q(x, \xi)$ par rapport à $\left.\xi\right)$.

Pour la démonstration du lemme, voir Lemma 3 dans [3].

Supposons qu'un opérateur $P(x, D)$ vérifie la condition exposée dans le théorème précédent. Soit $\Omega^{\prime}$ un ouvert borné, $\bar{\Omega}^{\prime} \subset \Omega$. D'après [1], nous pouvons nous affirmer que pour tout $x \in \Omega$ il existe des constantes positives $A_{x}$. et $C_{x}$ telles que

$$
\left\{\begin{array}{l}
\tilde{P}(x, \xi) \leqslant 2|P(x, \xi)| \\
P(x, \xi) \neq 0 \\
1 /|P(x, \xi)| \leqslant C_{x}(1+|\xi|)^{m} x
\end{array}\right.
$$

pour tout $\xi$ vérifiant $|\xi| \geqslant A_{x}$, oú $A_{x}$ et $C_{x}$ sont bornés quand $x$ parcourt des ensembles compacts de $\Omega$ et $m_{x}=$ l'order de $P(x, \xi)$ par rapport à $\xi$. 
Posons

$$
A=\max _{x \in \Omega^{\prime}} A_{x}, \quad m=\max _{x \in \Omega^{\prime}} m_{x}
$$

et

$$
a^{\prime}=\min _{|\xi| \leqslant A, y \in \Omega^{\prime}}\left(a \tilde{P}(y, \xi) /(1+|\xi|)^{m}\right)>0 .
$$

Compte tenu de (2.1), le lemme précédent peut être appliqué pour $Q(x, D)$ $=P(x, D)$ et $K=\bar{\Omega}^{\prime}$ : pour $\xi_{0}$ vérifiant $\left|\xi_{0}\right| \leqslant A$ et pour $y_{0} \in \Omega^{\prime}$, il existe $\theta_{0} \in R^{n}$ tel que

$$
b \widetilde{P}(y, \xi) \leqslant \inf _{|z|=1}\left|P\left(y, \xi+z \theta_{0}\right)\right|
$$

pour $\xi$ et $y$ vérifiant l'inegalité

$$
\left|\xi-\xi_{0}\right| \leqslant \eta, \quad\left|y-y_{0}\right| \leqslant a^{\prime} .
$$

Maintenant nous prenons $\xi^{(1)}, \ldots \ldots, \xi^{(r)} \in R^{n}$ dans $|\xi| \leqslant A$ et $y^{(1)}, \ldots \ldots, y^{(s)} \in R^{n}$ dans $\Omega^{\prime}$ tels que deux familles d'ensembles $\left\{\xi ;\left|\xi-\xi^{(p)}\right| \leqslant \eta\right\}_{p=1}^{r}$ et $\left\{y ;\left|y-y^{(q)}\right|\right.$ $\left.\leqslant a^{\prime}\right\}_{q=1}^{s}$ soient des recouvrement de $\{\xi ;|\xi| \leqslant A\}$ et $\Omega^{\prime}$ respectivement. Alors nous choisissons des fonctions $0 \leqslant \varphi_{p}(\xi) \in \mathscr{D}(p=1, \ldots \ldots, r)$ et $0 \leqslant h_{q}(y) \in \mathscr{D}$ $(q=1, \ldots \ldots, s)$ de la manière suivante:

$$
\begin{aligned}
& \operatorname{Supp}\left[\varphi_{0}\right] \subset\{\xi ;|\xi| \geqslant A\}, \\
& \operatorname{Supp}\left[\varphi_{p}\right] \subset\left\{\xi ;\left|\xi-\xi^{(p)}\right| \leqslant \eta\right\} \quad(p=1, \ldots \ldots, r), \\
& \operatorname{Supp}\left[h_{q}\right] \subset\left\{y ;\left|y-y_{q}\right| \leqslant a^{\prime}\right\} \quad(q=1, \ldots \ldots, s), \\
& \varphi_{0}(\xi)+\ldots \ldots+\varphi_{r}(\xi)=1 \quad \text { dans } R^{n}, \\
& h_{1}(y)+\ldots \ldots+h_{s}(y)=1 \quad \text { dans } \Omega^{\prime} .
\end{aligned}
$$

Par $\theta_{p, q}$ nous désignons $\theta$ correspondant à $\xi^{(p)}$ et $y^{(q)}$ dans le lemme, et par $E_{0}(x, y)$ et $E_{p, q}(x, y)$ des distributions sur $R_{x}^{n}$ définies pour chaque $y \in \Omega^{\prime}$ comme suit $(p=1, \ldots \ldots, r ; q=1, \ldots \ldots, s)$ :

$$
\left\langle E_{0}(x, y), \quad \check{\phi}(x)\right\rangle=(2 \pi)^{-n} \int \frac{\varphi_{0}(\xi)}{P(y, \xi)} \hat{\phi}(\xi) d \xi, \quad \phi \in \mathscr{D},
$$

(2. 4) $\left\langle E_{p, q}(x, y), \check{\phi}(x)\right\rangle$

$$
=(2 \pi)^{-n} \int\left(\int_{|z|=1} \frac{\varphi_{q}(\xi) h_{q}(y)}{P\left(y, \xi+z \theta_{q, p}\right)} \hat{\phi}\left(\xi+z \theta_{p, q}\right) \frac{1}{2 \pi i z} d z\right) d \xi, \quad \phi \in \mathscr{D},
$$

ici

$$
\hat{\phi}(\xi)=\mathscr{F}[\phi](\xi)=\int e^{-i x \xi} \phi(x) d x, \quad \check{\phi}(x)=\phi(-x) .
$$


On déduit sans difficulté de (2.1) et (2.2) qu'elles sont bien définies, et en outre, de (2.3) que $E_{0}(x, y) \in \mathcal{E}_{y}(\Omega)\left(\mathscr{S}_{x}^{\prime}\right)$ et

$$
\hat{E}_{0}(\xi, y)=\mathscr{F}_{x}\left[E_{0}(x, y)\right]=\frac{\varphi_{0}(\xi)}{P(y, \xi)} .
$$

De plus on déduit de (1.2)

$$
E_{0}(x, y) \in \mathcal{E}_{y}\left(\Omega^{\prime}\right)\left(k ; A^{-(m+k), d}\right),
$$

( $k$ et $d$ ont la même signification qu'en (H. 2)), et de (2. 4)

$$
E_{p, q}(x, y)=(2 \pi)^{-n} \int\left(\int_{|z|=1} \frac{\varphi_{p}(\xi) h_{q}(y)}{P\left(y, \xi+z \theta_{p, q}\right)} e^{i x\left(\xi+z \theta_{p, q}\right)} \frac{1}{2 \pi i z} d z\right) d \xi,
$$

ce qui montre $E_{p, q}(x, y) \in C^{\infty}\left(R_{x}^{n} \times \Omega^{\prime}\right)$.

Proposition. Supposons que $P(x, D)$ soit le même qu'en théorème et $E_{0}(x, y)$ et $E_{p, q}(x, y)$ soient les distributions définies par (2.3) et (2.4) respectivement. Alors la distribution

$$
E(x, y)=E_{0}(x, y)+\sum_{p=1}^{r} \sum_{q=1}^{s} E_{p, q}(x, y)
$$

sur $R_{x}^{n}$ est une solution élémentaire de $P(y, D)$ pour chaque $y \in \Omega^{\prime}$ et appartient $\grave{a}$ $\varepsilon_{y}\left(\Omega^{\prime}\right)\left(k ; A^{-(m+k), d}\right)$.

Démonstration. Soit $\varphi \in \mathscr{D}$. Alors on déduit de (2.3), (2.4) et de la formule d'inversion de Fourier,

$$
\begin{array}{r}
\left\langle P\left(y, D_{x}\right) E(x, y), \quad \check{\phi}(x)\right\rangle=\left\langle E(x, y), P\left(y, D_{x}\right) \phi\right\rangle \\
=(2 \pi)^{-n} \sum_{p=0}^{r} \sum_{q=1}^{s} \int \varphi_{p}(\xi) h_{q}(y) \hat{\varphi}(\xi) d \xi=\varphi(0),
\end{array}
$$

pour $y \in \Omega^{\prime}$. D'ou résulte $P\left(y, D_{x}\right) E(x, y)=\delta(x)$ pour $y \in \Omega^{\prime}$. D'autre part on a $E(x, y) \in \mathcal{E}_{y}\left(\Omega^{\prime}\right)\left(k, A_{1 \mathrm{oc}}^{-(m+k), d}\right)$ d'après $(2.6)$ et $(2.7)$.

Cela achève la démonstration de la proposition.

3. Soit $P(x, D)$ un opérateur différentiel à coefficients indéfiniment dérivables dans un ouvert $\Omega$ de $R^{n}$ et $\Omega^{\prime}$ un ouvert borné, $\bar{\Omega}^{\prime} \subset \Omega$. Supposons que $P(x, D)$ vérifie la condition HE dans $\Omega$. Alors nous allons démontrer qu'il est de type progressif dans $\Omega^{\prime}$.

Nous avons déjà vu dans le paragraphe précédent que $P(x, D)$ possède la propriété (T. 1). Il suffit donc de démontrer que $P(x, D)$ possède la propriété 
(T. 2). Pour cela nous pouvons supposer que les coefficients de $P(x, D)$ appartiennent à $\mathscr{D}(\Omega)^{1}$ ):

$$
P(x, D)=\sum_{|\alpha| \leqslant m} b_{\alpha}(x) D^{\alpha}, \quad b_{\alpha}(x) \in \mathscr{D}(\Omega) .
$$

Soient $E(x, y), E_{0}(x, y)$ et $E_{p, q}(x, y)(p=1, \ldots \ldots, r ; q=1, \ldots \ldots, s)$ des distributions définies par (2.3), (2.4) et (2.8), $\Omega^{\prime \prime}$ un ouvert borné, $\bar{\Omega}^{\prime \prime} \subset \Omega^{\prime}$, et $\omega(x)$ une fonction appartenant à $\mathscr{D}$, de support contenu daus $\left\{x ; x+\Omega^{\prime \prime} \subset \Omega^{\prime}\right\}$ et égale à 1 au voisinage de 0 . Nous désignons alors par $f_{j}(x, y)$ et $g_{j}(x, y)(j=0,1, \ldots \ldots)$ des distributions sur $R_{x}^{n}\left(y \in \Omega^{\prime \prime}\right)$ qui sont déterminées par

$$
\left\{\begin{aligned}
f_{0}(x, y) & =E_{0}(x, y) \\
g_{0}(x, y) & =\sum_{p=1}^{r} \sum_{q=1}^{s} E_{p, q}(x, y) \\
f_{j}(x, y) & =f_{0}(x, y)_{(x)}^{*}\left[\omega(x)\left\{P\left(y, D_{x}\right)-P\left(x+y, D_{x}\right)\right\} f_{j-1}(x, y)\right] \\
g_{j}(x, y) & =g_{0}(x, y)_{(x)}^{*}\left[\omega(x)\left\{P\left(y, D_{x}\right)-P\left(x+y, D_{x}\right)\right\}\left(f_{j-1}(x, y)+g_{j-1}(x, y)\right)\right] \\
& +f_{0}(x, y)_{(x)}^{*}\left[\omega(x)\left\{P\left(y, D_{x}\right)-P\left(x+y, D_{x}\right\} g_{j-1}(x, y)\right],(j \geqslant 1) .\right.
\end{aligned}\right.
$$

On voit facilement que $T_{j}(x, y)=f_{j}(x, y)+g_{j}(x, y)$ vérifie (1.1) pour $y \in \Omega^{\prime \prime}$. Pour examiner la propriété (T. 2) nous n'avons qu' à démontrer que pour tout $q \in N$ il existe un $p \in N$ tel que $f_{p}(x, y)+g_{p}(x, y) \in C^{q}\left(R_{x}^{n} \times \Omega_{y}^{\prime \prime}\right)$.

D'après (2.7) et la proposition, on a $g_{0}(x, y) \in C^{\infty}\left(R_{x}^{n} \times \Omega^{\prime \prime}\right)$ et $f_{0}(x, y) \in \mathcal{E}_{y}\left(\Omega^{\prime \prime}\right)\left(\mathscr{D}_{x}^{\prime}\right)$ respectivement. Donc, en utilisant le fait que

$$
\begin{aligned}
& C^{\infty}\left(R_{x}^{n} \times \Omega_{y}^{\prime \prime}\right)_{(x)}^{*} \mathcal{E}_{y}\left(\Omega^{\prime \prime}\right)\left(\mathcal{E}_{x}^{\prime}\right) \subset C^{\infty}\left(R_{x}^{n} \times \Omega_{y}^{\prime \prime}\right)^{2)}, \\
& \mathcal{E}_{y}\left(\Omega^{\prime \prime}\right)\left(\mathscr{D}_{x}^{\prime}\right)_{(x)}^{*} C_{0}^{\infty}\left(R_{x}^{n} \times \Omega_{y}^{\prime \prime}\right) \subset C^{\infty}\left(R_{x}^{n} \times \Omega_{y}^{\prime \prime}\right),
\end{aligned}
$$

on déduit de (3.1) $g_{j}(x, y) \in C^{\infty}\left(R_{x}^{n} \times \Omega_{y}^{\prime \prime}\right)$ pour tout $j \geqslant 0$.

Maintenant on va démontrer que pour $\mu, \nu \in N^{n}$ et $j \geqslant 0$ il existe une constante $C_{\mu, \nu, j}$ telle que

$$
\left|D_{\xi}^{\mu} D_{y}^{\nu} \hat{f}_{j}(\xi, y)\right| \leqslant C_{\mu, \nu, j}(1+|\xi|)^{-d\left(\mid \mu_{1+j}\right)} M^{\nu-\mu}(y, \xi) / \widetilde{P}(y, \xi)
$$

pour tout $\xi \in R^{n}$ et $y \in \Omega^{\prime \prime}$, ici $d$ et $M(y, \xi)$ sont la même signification qu'en (H. 2).

Si $f_{j}(x, y)(j \geqslant 0)$ vérifieront (3.2), on déduit de (1.2) et (2.1) que pour $\xi \in R^{n}$ et $y \in \Omega^{\prime \prime}$

1) Soit $\beta(x) \in \mathscr{D}(\Omega)$ et égale à 1 sur $\Omega^{\prime}$. Considérons alors l'opérateur $\beta(x) P(x, D)$ à la place de $P(x, D)$.

2) Nous désignons par $\mathcal{E}^{\prime}$ l'espace des distributions à support compact. 


$$
\begin{aligned}
& \left|\mathscr{F}_{x}\left[x^{\mu} D_{y}^{\nu} f_{j}(x, y)\right]\right|=\left|D_{\xi}^{\mu} D_{y}^{\nu} \hat{f}_{j}(\xi, y)\right| \\
& \leqslant C(1+|\xi|)^{-d\left(\mid \mu_{1+j}\right)} M^{\nu-\mu}(y, \xi) / \tilde{P}(y, \xi) \\
& \leqslant C(1+|\xi|)^{-d\left(\mid \mu_{1+j}\right)}(1+|\xi|)^{k_{1 \nu 1}}(1+|\xi|)^{m},
\end{aligned}
$$

ce qui montre $f_{j}(x, y) \in \mathcal{E}_{y}\left(\Omega^{\prime \prime}\right)\left(k ; A^{-(m+k)+d j, d}\right)$. Or on peut s'affirmer que pour tout $q \in N$ il existe un $p \in N$ tel que $\varepsilon_{y}\left(\Omega^{\prime \prime}\right)\left(k ; A^{-(m+k)+d p, d}\right) \subset C^{q}\left(R_{x}^{n} \times \Omega_{y}^{\prime \prime}\right)$, donc $P(x, D)$ possède la propriété (T. 2), ce qui achève la démonstration du théorème, comme $\Omega^{\prime \prime}$ est un ouvert borné arbitraire tel que $\bar{\Omega}^{\prime \prime} \subset \Omega^{\prime}$.

On démontrera la relation (3.2) par induction. Pour cela on n'a qu'à montrer

(a) si la relation (3. 2) est vraie pour tout $j^{\prime}<j$ et tout $\nu, \mu$, elle est vraie pour $j$, $\mu=0$ et $\nu=0$,

(b) si la relation (3.2) est vraie pour tout $j^{\prime} \leqslant j$ et tout $\nu^{\prime}, \mu^{\prime}$ tels que $\left|\nu^{\prime}+\mu^{\prime}\right|<|\nu+\mu|$, elle est vérifiée pour $j, \nu$ et $\mu$.

Démonstration de $(a)$. On pose

$$
\begin{aligned}
& f_{j}(x, y)=f_{0}(x, y)_{(x)}^{*}\left\{P\left(y, D_{y}\right)-P\left(x+y, D_{x}\right)\right\} f_{j-1}(x, y) \\
& \quad-f_{0}(x, y)_{(x)}^{*}\left[(1-\omega(x))\left\{P\left(y, D_{x}\right)-P\left(x+y, D_{x}\right)\right\} f_{j-1}(x, y)\right] .
\end{aligned}
$$

D'après le développement de Taylor, on a:

$$
P\left(y, D_{x}\right)-P\left(x+y, D_{x}\right)=\sum_{0<|\alpha|<t} C_{\alpha} x^{\alpha} D_{y}^{\alpha} P\left(y, D_{x}\right)+\sum_{|\alpha|=t} x^{\alpha} R_{\alpha}\left(x, y ; D_{x}\right\rangle,
$$

avec des constantes $C_{\alpha}$ convenables, $R_{\alpha}\left(x, y ; D_{x}\right)$ étant un opérateur différentiel s'écrivant sous la forme

$$
R_{\alpha}\left(x, y ; D_{x}\right)=\sum_{|\gamma| \leqslant m} c_{\alpha, \gamma}(x, y) D_{x}^{\gamma},
$$

ici on note que chaque fonction $c_{\alpha, \gamma}(x, y)$ est indéfiniment dérivable dans $R_{x}^{n} \times R_{y}^{n}$ et bornée ainsi que chacune de ses dérivées.

Par conséquent, grâce à (2. 5), (3. 3) et (3.4) on a:

$$
\begin{aligned}
& P(y, \xi) \hat{f}_{j}(\xi, y)=\varphi_{0}(\xi) \sum_{0<|\alpha|<t} C_{\alpha}\left(-D_{\xi}\right)^{\alpha}\left(D_{y}^{\alpha} P(y, \xi)\right) \hat{f}_{j-1}(\xi, y) \\
& \quad+\varphi_{0}(\xi) \sum_{|\alpha|=t} \sum_{|\gamma| \leqslant m} \mathscr{F}_{x}\left[x^{\alpha} c_{\alpha, \gamma}(x, y) D_{x}^{\gamma} f_{j-1}(x, y)\right] \\
& \quad+\varphi_{0}(\xi) \mathscr{F}_{x}\left[(1-\omega(x)) \sum_{|\gamma| \leqslant m}\left(b_{\gamma}(y)-b_{\gamma}(x+y)\right) D_{x}^{\gamma} f_{j-1}(x, y)\right]
\end{aligned}
$$

D'abord nous considérons le premier terme du second membre de (3.5): d'aprèe (1.2) et la hypothèse de l'induction, il existe une constante $C_{\alpha, j}>0$ telle que 


$$
\begin{aligned}
& \left|D_{\xi}^{\beta} D_{y}^{\alpha} P(y, \xi) D_{\xi}^{\alpha-\beta} \hat{f}_{j-1}(\xi, y)\right| \\
& \quad \leqslant C_{\alpha, j}(1+|\xi|)^{-d|\beta|} M^{\alpha-\beta}(1+|\xi|)^{-d(|\alpha-\beta|+j-1)} M^{-(\alpha-\beta)} \\
& \quad \leqslant C_{\alpha, j}(1+|\xi|)^{-d j}, \quad(\forall \beta \leqslant \alpha, \quad \alpha \neq 0)
\end{aligned}
$$

pour tout $\xi \in R^{n}$ et $y \in \Omega^{\prime \prime}$.

Maitenant pour le second terme: d'abord on remarque qu'il existe une constante $C_{t, j}>0$ telle que pour tout $\alpha(|\alpha|=t), \gamma$ et $y$,

$$
\int\left|\mathscr{F}_{x}\left[\frac{c_{\alpha, \gamma}(x, y)}{\left(1+|x|^{2}\right)^{n}}\right](\xi)\right|(1+|\xi|)^{d j} d \xi \leq C_{t, j}
$$

et on déduit de (2. 1) et de la hypothèse de l'induction que

$$
\left|\left(1+\Delta_{\xi}\right)^{n} D_{\xi}^{\alpha}\left(\xi^{\gamma} \hat{f}_{j-1}(\xi, y)\right)\right| \leqslant C_{j}(1+|\xi|)^{-d j}, \xi \in R^{n}, y \in \Omega^{\prime \prime}
$$

pour $\alpha,|\alpha|=t_{0}\left(t_{0}=[(2 m+d) / d]+1, \quad \Delta_{\xi}=-\sum_{j=1}^{n} \frac{\partial^{2}}{\partial x_{j}{ }^{2}}\right) . \quad$ On a alors:

$$
\begin{aligned}
\operatorname{Sup}_{\xi \in R^{n}} & \mathscr{F}_{x}\left[x^{\alpha} c_{\alpha, \gamma}(x, y) D_{x}^{\gamma} f_{j-1}(x, y)\right](1+|\xi|)^{d j} \\
& \leqslant(2 \pi)^{-n} C_{t_{0}, j} \sup _{\xi \in R^{n}}\left|\left(1+\Delta_{\xi}\right)^{n} D_{\xi}^{\alpha}\left(\xi^{\gamma} \hat{f}_{j-1}(\xi, y)\right)\right|(1+|\xi|)^{d j} \\
& \leqslant(2 \pi)^{-n} C_{t_{0}, j} C_{j} .
\end{aligned}
$$

pour $\alpha,|\alpha|=t_{0}$.

Enfin pour le troisième terme: comme $1-\omega(x)=0$ au voisinage de 0 , on a

$$
\begin{aligned}
\mathscr{F}_{x} & {\left[(1-\omega(x))\left(b_{r}(y)-b_{\gamma}(x+y)\right) D_{x}^{\gamma} f_{j-1}(x, y)\right] } \\
& =(2 \pi)^{-n} \mathscr{F}_{x}\left[\frac{1-\omega(x)}{|x|^{2 s}}\left(b_{\gamma}(y)-b_{\gamma}(x+y)\right)\right](\xi)_{(\xi)}^{*} \Delta_{\xi}^{s}\left(\xi^{\gamma} \hat{f}_{j-1}(\xi, y)\right),
\end{aligned}
$$

où $s \geqslant \max (n,(2 m+d) / 2 d)$.

Alors par le même raison qu'en (3.6), il existe une constante $C_{s, j}>0$ telle que

$$
\left|\mathscr{F}_{x}\left[(1-\omega(x))\left(b_{\gamma}(y)-b_{r}(x+y)\right) D_{x}^{r} f_{j-1}(x, y)\right]\right| \leqslant C_{s, j}(1+|\xi|)^{-d j}
$$

pour tout $\xi \in R^{n}$ et tout $y \in \Omega^{\prime \prime}$. Cela termine la démonstration de (a).

Démonstration de $(b)$. En opérant $D_{\xi}^{\mu} D_{y}^{\nu}$ à (3. 5), on a :

$$
P(y, \xi) D_{\xi}^{\mu} D_{y}^{\nu} \hat{f}_{j}(\xi, y)
$$

$$
=\sum_{\substack{\nu^{\prime}+\nu^{\prime \prime}=\nu \\ \mu^{\prime}+\mu^{\prime \prime}=\mu \\ \nu^{\prime}+\mu^{\prime}>0}} C_{\nu^{\prime}, \mu^{\prime}}\left(D_{\xi}^{\mu \prime} D_{y}^{\nu \prime} P(y, \xi)\right) \cdot\left(D_{\xi}^{\mu \prime \prime} D_{y}^{\nu \prime \prime} \hat{f}_{j}(\xi, y)\right)
$$


(ii)

$$
\begin{aligned}
& +\sum_{\substack{\nu^{\prime}+\nu^{\prime \prime}=\nu \\
\mu^{\prime}+\mu^{\prime \prime}=\mu}} \sum_{0<|\alpha|<t} C_{\alpha, \nu^{\prime}, \mu^{\prime}} D_{\xi}^{\alpha}\left[D_{\xi}^{\mu \prime} D_{y}^{\nu \prime+\alpha}\left(P(y, \xi) \varphi_{0}(\xi)\right) D_{\xi}^{\mu \prime \prime} D_{y}^{\nu \prime \prime} \hat{f}_{j-1}(\xi, y)\right] \\
& \text { (iii) } \quad+\sum_{|\alpha|=t} \sum_{|r| \leqslant m} D_{\xi}^{\mu} D_{y}^{\nu}\left(\mathscr{F}_{x}\left[x^{\alpha} c_{\alpha, \gamma}(x, y) D_{x}^{\gamma} f_{j-1}(x, y)\right] \varphi_{0}(\xi)\right) \\
& \text { (iv) } \quad+D_{\xi}^{\mu} D_{y}^{\nu}\left(\varphi_{0}(\xi) \mathscr{F}_{x}\left[(1-\omega(x)) \sum_{|r| \leqslant m}\left(b_{\gamma}(y)-b_{\gamma}(x+y)\right) D_{x}^{\gamma} f_{j-1}(x, y)\right]\right) \text {. }
\end{aligned}
$$

Nous allons estimer (i), (ii), (iii) et (iv) par $\xi$.

D'abord on déduit facilement de (1.2) et de la hypothèse de l'induction qu'il existe une constance $C_{\mu, \nu, j}>0$ telle qu'aucun terme de (i) et (ii) ne dépasse $C_{\mu, \nu, j}(1+|\xi|)^{-d(|\mu|+i)} M(y, \xi)^{\nu-\mu}$ pour tout $\xi \in R^{n}$ et $y \in \Omega^{\prime \prime}$.

Du meme calcul qu'en (3.6) on a:

$$
\begin{aligned}
\mid D_{\xi}^{\mu} D_{y}^{\nu} \mathscr{F}_{x}[ & \left.x^{\alpha} c_{\alpha, \gamma}(x, y) D_{x}^{\gamma} f_{j-1}(x, y)\right] \mid \\
& \leqslant C_{\mu, \nu, j}(1+|\xi|)^{-d(|\mu|+i)} M(y, \xi)^{\nu-\mu}
\end{aligned}
$$

si $|\alpha|=t_{0}\left(t_{0}=[(2 m+d+k|\nu+\mu|) / d]+1\right)$. En appliquant à (iv) la méthode semblable qu'en haut, on peut obtenir la démonstration de (b).

\section{BiBLIOGRAPHIE}

[1] L. Hörmander: Hypoelliptic differential operators, Ann. Inst. Fourier. Grenoble 11 (1961), 477-492.

[2] F. Trèves: Opérateurs différentiels hypoelliptiques, Ann. Inst. Fourier. Grenoble 9 (1959), $1-73$.

[3] F. Trèves: Fundamental solutions of linear partial differential equations with constant Coefficients depending on parameters, Amer. J. Math. 84 (1962), 561-577.

Nagoya University 